\title{
THE LEAR CONTROL SYSTEM - PHASE II
}

\author{
Michel CHANEL and Thomas PETTERSSON \\ CERN, CH-1211 Geneva 23, Switzerland
}

The LEAR control system and its current status are described briefly. The system consists today of two interconnected PDP 11/73's and one microVAX in an Ethernet-like local area network. For real-time requirements, CAMAC-based microprocessor systems are used. The main point of the paper is the description of an evolutive data base architecture where the emphasis is on access to accelerator physics parameters rather than to power supply parameters.

\section{Introduction}

The control system of the LEAR machine [1] furnishes a stable, reliable base upon which higher levels of application and system-related software are now being built.

The new software must be structured in such a fashion that three seemingly opposing goals can be met: the system should provide a tool to adapt the accelerator to the ever increasing requirements for new operational modes from the physics experiments [2]; the system should require a minimum of manpower support in production and, last but not least, the system should be user-friendly.

This implies that the control system must evolve to encompass all instrumentation that is now manually operated (such as spectrum analyzers, digital oscilloscopes, etc.), acquire a higher degree of machine fault or malfunction detection, as well as becoming a real tool for machine development studies.

The accelerator has a working range from approximately $100-2000 \mathrm{MeV} / \mathrm{c}$ using protons, antiprotons and $\mathrm{H}^{-}$ions; the particle momentum levels as shown in fig. 1 represent different stages in the process of preparing the beam for the ultraslow ejection (currently one hour). The beam is extracted and transported to the experiment stations by a sophisticated beam switchyard with two splitters to provide three experiments simultaneously with cooled antiprotons [3].

\section{The basic control system}

The basic control system is described in detail in ref. [1] and only a short overview is given here.

The control system is based on two PDP 11/73 microcomputers and one microVAX computer in a network (fig. 2). The network hardware consists of a mixture of Ethernet-like and Ethernet modules and the software is also a mixture of DECnet-like software and DECnet. One of the microcomputers is connected to the hardware to be controlled through a combination of parallel and serial-loop connected CAMAC, as shown.

An important component of the control system is known as the GFA which is the French acronym for an autonomous function generator. A number of these hardware modules, with the associated timing system [4] enables us to generate the type of machine cycle shown in fig. 1, by making the power supplies follow a series of vectors as a function of time.

GFAs are used to control the main elements (quadrupoles, sextupoles and dipoles) and the rf voltage programs.

The vectors for the GFAs are generated in the GFA microcomputer and down-loaded through CAMAC.

To create and change the machine cycle according to operational requirements, a powerful editing system for the GFAs [5] is available. The editor may be used both interactively with a graphics screen, or by another program issuing commands for function modifications.

In the same spirit, another tool has been developed for the "scanning mode" [6]. This is the procedure of adjusting the particle momentum between two wellknown levels by a nearly-linear interpolation of the GFA function values at any given flat-top in the cycle. This change of the length of the vectors that describe the machine cycle also requires a change of the associated timing events that synchronizes all the GFAs.

For energy-dependent parameters not controlled by a GFA, the real-time requirements for switching the hardware as a function of the timing system interrupts are handled by local microprocessors. Other timing interrupt functions, where the real-time requirements are more relaxed, are handled in the control computer.

The process access software is based on a database philosophy, with the online database situated in an external CAMAC module. User programs submit access requests to the database or the hardware using the 

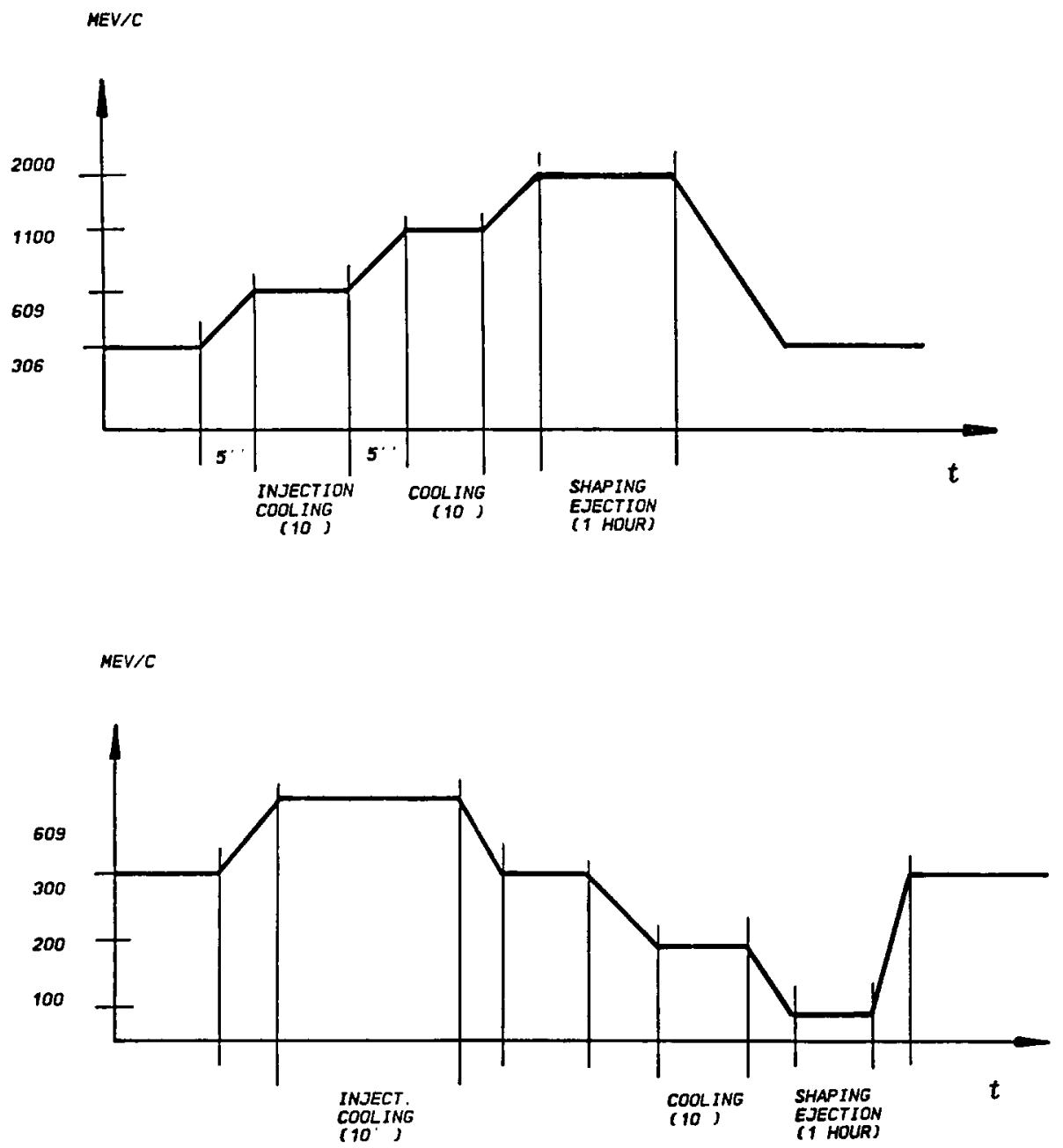

Fig. 1. Example of LEAR cycles.

standard operating system calls that are routed to the data base manager task (DBM). Each addressable parameter in the system is known by a unique name, and this name is used for all accesses, whether the parameter is a simple 16-bit word or a 64 element vector for a GFA.

Operators interact with the system from five generalized consoles that use the now classical touch-panel, colour TV, numerical keypad and shaft encoders to transmit commands and receive information. These commands include controlling a parameter by the shaft encoder, starting synoptic displays, having a log printed, or executing an application program.

Currently, there is only one of the computers that is connected to the CAMAC hardware and consequently all access from the two others have to pass via the network. There is a slight time delay penalty in execution to pass across the network, but this is within acceptable limits.

\section{Present problems}

Each GFA may supply up to 64 different function values during the cycle. Thus, to change the beam characteristics, for whatever purpose, may involve the modification and reloading of more than 80 GFAs.

However, modifying the function generator data nearly always implies also changing the timing data. The timing system controls the evolution of the magnetic cycle and also the remainder of equipment that is energy dependent and thus related to given instants the machine cycle. Changing a GFA almost invariably entails changing the timing of the cycle, which requires a reload of the master timing generator.

No scanning program is yet available for various subsystems since they do not have the simple linear relation to the beam energy as the power supplies do, requiring more complex calculations (such as stochastic cooling or experimental beam splitting etc.). 
CONSOLES

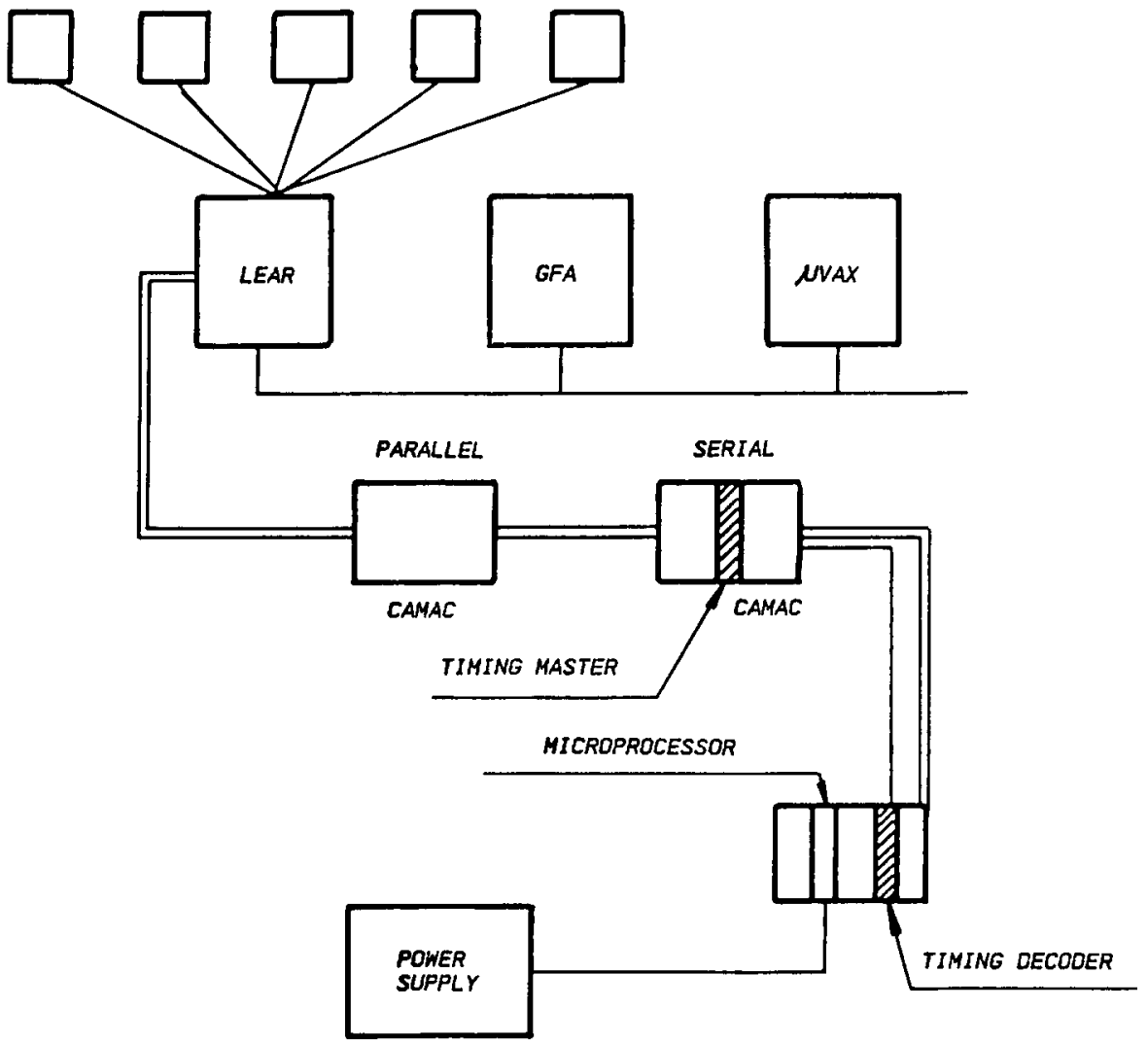

Fig. 2. Principle of the hardware layout.

The current approach to parameter modifications, due momentum adjustment has been on an as needed basis as the machine is being set up and tuned, requiring the presence of the specialists for each subsystem. Once the machine is thus tuned, the state is archived for later retrieval.

The need for a more powerful software system becomes evident when the physics program requires six different energy levels in as many days and that currently a new energy takes roughly $12 \mathrm{~h}$ to set up.

Obviously, once all energy levels have been covered and the covered and the corresponding machine states have been archived, setting-up will be done in much shorter time. However, this pleasant prospect does not seem likely to be achieved, since the machine and the switchyard is in permanent evolution, changing the working conditions constantly.

Another requirement from the user community is to change the energy level from pulse to pulse, a change of a few percent of the momentum, which may or may not influence other subsystems.

The implications on the possible future operation of the machine with an internal target must also be considered in the context of the control system.

\section{Proposal for improvements}

There is a number of machine parameters whose values are momentum/time dependent, e.g. the stochastic cooling systems, the ultraslow ejection system and the machine tune. Currently these parameters are treated by individual programs that allow the editing and manipulation of the parameter values for each energy in question.

To implement a standard interface to these multivalued types of parameters, we propose to add a second data base using the facilities of the current single-value data base for hardware access and operational use.

A multivalued parameter may be considered as a "GFA" type of parameter with an arbitrary number of function values. This has the added advantage that the GFAs, currently handled by separate data base system, may be integrated into the new data base and treated in the same fashion as any other time/momentum-dependent parameters. The editing of the parameter function values should be done in the same fashion, regardless of what kind of system the parameter belongs to. The editing should be done in either the interactive mode or 
noninteractively by other programs.

Since the real-time requirements are handled by local microprocessors, the new data base may be allowed to have a much more complex internal structure without any performance penalties for the operation of the accelerator and the beam line switchyard.

The data base must also be able to handle more complex parameter relationships, e.g. changing and downline loading a given parameter may require changing and downline loading other parameters in the control system.

Machine physics parameters such as chromaticity, the machine tune, horizontal and vertical emittances should also be considered as parameters in the database, although they require the execution of a program to be accessed, both for acquisition and control. Currently, these parameters may be controlled and acquired but not in a system-integrated fashion, each requiring an explicit manual request for execution.

Depending on whether the accelerator is in high energy mode $(>1.0 \mathrm{GeV} / c)$, in low energy mode $(<1.0$ $\mathrm{GeV} / c)$ or in ultralow energy mode $(<0.2 \mathrm{GeV} / c)$, many parameters require rescaling of the engineering units used.

The LEAR uses an important amount of HPIBinterfaced instrumentation that has to be computer controlled. Each HPIB controller CAMAC module is in turn controlled by a local microprocessor which handles the real-time part of the instrumentation control. These instruments should also be considered as having timedependent parameters with single or multiple values (e.g. the settings of the spectrum analyzers change with the momentum level and should thus be under computer control when in production to ensure correct settings at any instant).

The editing program for this data base has to be able to execute on any of the control system computers without regard to where an actual parameter is situated.

The new database programs will probably include:

1) a Data Base Manager, handling access to data storage and to the lower level data base;

2) a database editing program having several levels of sophistication, depending on the host computer system (PDP11 or VAX);

3) an access program to allow remote access from other computers.

\section{The database programs}

The Extended Data Base Manager (DBX) is the program (or programs) that handles the execution of requests from the data base editor or other online programs. The DBX presents a well defined interface to the user and covers the lower-level details of hardware and remote computer access. The interest of having one or a small number of programs doing the low level system and hardware-oriented work for all users is obvious in terms of security, programming efficiency and resource usage.

Fig. 3 shows how the online programs interact with the different control system components today and fig. 4 shows how the future interaction will look.

One aim of the new system is to improve the overall useability and to let the accelerator people concentrate on their main task: to make the accelerator function and to not waste more time on details of computer programming than strictly necessary.

The DBX must be able to absorb the addition of new complex subsystems without major surgery and without doing one-off solutions for problems that do not fit into a preconceived concept, and it must be capable of handling a number of different relations between two arbitrarily selected entities. These include:

(a) Parameter to CAMAC module in the local system. This is the simplest type of relation and is in principle already implemented by the low level data base. The relation consists of a parameter name linked to a hardware descriptor that indicates CAMAC addresses, calibration values, minimum/maximum values, etc. There is only one control value (if any), but there might be several acquisition values (such as multiwire proportional chamber parameters).

(b) Parameter to CAMAC module in a remote system. This relation allows programs to access remote parameters as if they were located in the host system. The DBX must cover the remote access procedure.

(c) Parameter-to-program in the local or remote system. This relation links a parameter to a program that has to be executed before the parameter itself may be evaluated (e.g. chromaticity or emittance). The DBX must be able to start a program, to survey its execution and to get the output result back to the caller. To avoid a duplication of programming effort, all programs that are liable to be used in this fashion should have two entries: one interactive and one noninteractive. The DBX must also be capable of starting a remotely-located program and to retrieve the resulting output.

A typical example of the latter case is the program that calculates the change in the tune value as a function of time. This program needs both information from the timing database as well as from the GFA database to be collected and transmitted to the system where the task is executing, by a task in the GFA/timing database computer.

(d) Parameter to microprocessor. This relation links the parameter to the address of a mailbox memory somewhere in the CAMAC loop or via the network, where the data is refreshed on a regular basis or to the request for the execution of a program to acquire and process the data before putting it in the common memory. A typical example concerns the digital oscilloscopes 


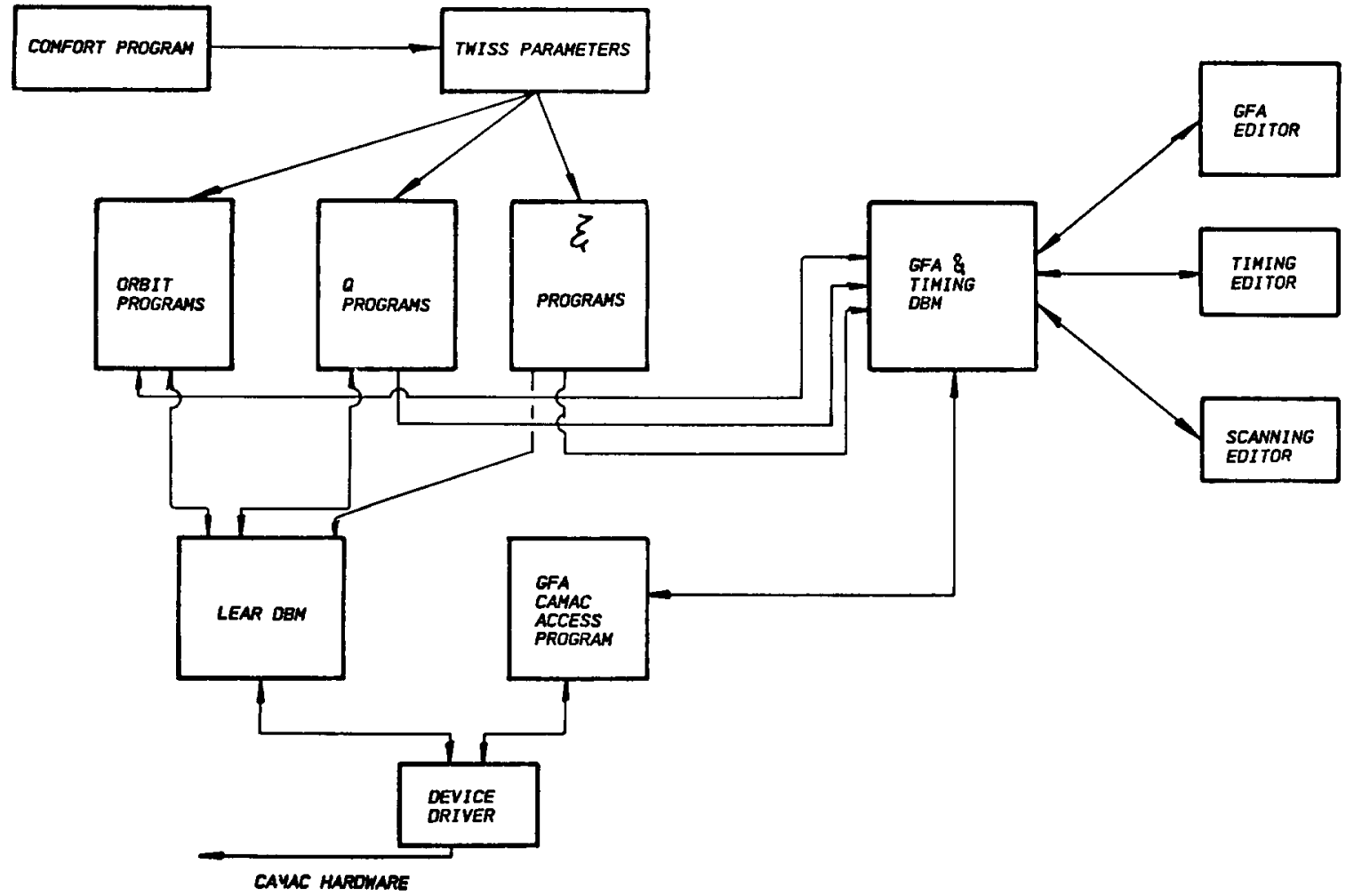

Fig. 3. Current software structure.

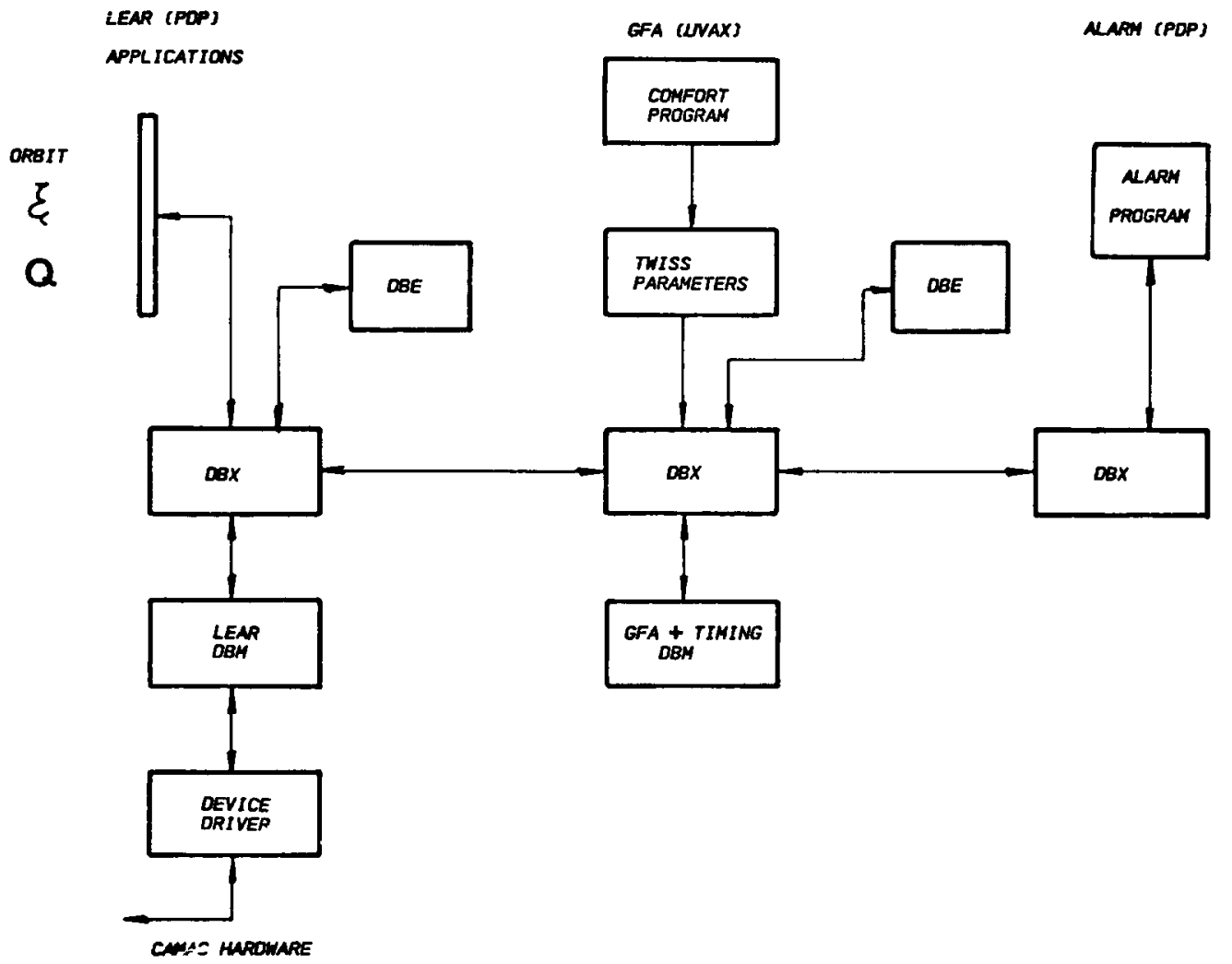

Fig. 4. Proposed software structure. 
used for surveillance of the injection. The data from these instruments are used to visualize the injection oscillations and also to calculate eventual corrections.

The DBX covers the actual hardware location of a parameter from the user, unless he insists, providing a transparent access for downline loading. This device independence allows modifications of the CAMAC hardware without rewriting of the application software which in our case presents a prohibitive exercise. A typical case is the replacement of the $15 \mathrm{yr}$ old GFAs (recuperated from the Booster accelerator) where no changes are needed at all in the application software.

The DBX provides the following functions:

(a) Downline load parameter. This function loads the hardware with the selected parameter value(s) and verifies by readback that the data has been properly received. A typical application is the downline loading of the GFAs, where the local microprocessor can interface with the load process if not properly synchronized with the timing.

(b) Access microprocessor-based parameter. This function covers the interaction with microprocessors and will mimic a standard parameter access on the user level. Access is either via CAMAC-based mailbox modules, direct access to the microprocessor memory or by using the Ethernet network which will support processto-process communication. The last mode of communication is also the most appealing from a programming standpoint.

(c) Compare/verify data base against hardware values. This function is mainly used at startup or when an error condition exists, i.e. no beam or beam lost. It verifies that the hardware control values actually are what they are thought to be. This function is a supplement to the alarm system.

\section{Database editor}

The Data Base Editor (DBE) must be capable of handling both multivalued parameters and the editing of the cycle timing data. For these functions, the current editors available will serve as starting points as concerns user/program interfacing and internal functionality.

The DBE should allow the nonspecialist, in normal operation of the machine, to modify any pertinent data in a user-friendly fashion. At the same time, it should provide means to access low level functions by the specialists, perhaps in a less user-friendly fashion. It should provide the following functions:

(a) Create parameter. This allows the creation of a parameter in the data base and verifies that the new parameter does not already exist somewhere in the system. This requires that an index of all parameters in available on all computer systems.

(b) Delete parameter. The opposite of the above function. The DBE must remove the parameter irrespectively of its location in the network.

(c) Enter/modify / extend relation. This function is one of the most important in the DBE. It should allow access to any kind of parameter defined, whether multivalued, microprocessor based or not. It will handle timing parameters, GFA parameters, etc., in an as closely related fashion as possible.

The multivalued parameters refer either to GFAs or to other momentum-dependent parameters. It must be possible to specify the timing instants when a given value is the current control value, to specify the sequence of values in either numerical form or from graphics input.

The timing system consists of a master clock capable of handling 256 events for a maximum duration of 128 h. This may seem a long time but the initial ultraslow ejection was calculated to be possible for only $15 \mathrm{~min}$, but today $1 \mathrm{~h}$ is normal [7]. The timing signal is distributed by a pulse-train to decoder modules, which may be enabled/disabled for a given event.

A cycle time of $24 \mathrm{~h}$ or more is foreseen later for stored-beam physics such as internal jet target, co-rotating $\mathrm{H}^{-} \mathrm{p}$ beams, etc. [8].

The DBE handles the setting of event timing, decoder enabling/disabling, the linking of one event to another, etc. The linking of events is important since changing the timing for an event in the beginning of the cycle may influence some or all subsequent events later in the cycle. It is necessary to know which event is related to which in this situation. For example, changing the timing for the deceleration to $309 \mathrm{MeV} / \mathrm{c} \mathrm{im}$ plies that all subsequent timing events must be updated but changing the timing for a measurement event does not change anything else.

\subsection{Local/remote database access}

The aim of the system is to provide as far as possible, transparent networking for the programming user of the control system. The DBX should be able to provide access to parameters in any computer system without forcing the user to be more than peripherally aware of this fact.

The network that is currently in use to connect the computers consists of a mixture of both hardware and software from different manufacturers. The medium term goal is to standardize on one hardware type (Ethernet) and one software product (DECnet). Being a small team, we must use as much externally supported software as possible.

The remote access for the data base will be handled by the local DBX that intercepts all calls to remote systems and returns the replies back to the user program. Each local DBX will have an index of what remote system has which parameter in its data base. 
This index is initialized at boot-time or when a parameter is either added or removed.

\subsection{Additional facilitıes}

The ability to use the output from already existing application programs as input to new programs is an important concept in our context. It allows for the construction of a chain of initially independent programs to form a new program entity providing entirely new capabilities, e.g. beam orbit calculations and corrections.

A number of large program systems for the calculation of machine parameters are available on the local computers in the control system, such as MiniAGS and the original version of COMFORT [9].

A new version of COMFORT developed at SLAC is also available over the CERNET network. The COMFORT program is used to calculate the Twiss parameters for a given nominal working point. The data are subsequently used in various applications as depicted in fig. 4.

Other well known computer codes used via the network are the MAD and DIMAT programs in the CERN computing centre.

\section{Programming languages}

The programming languages used in the system are manufacturer supported for obvious reasons and are:

(1) assembler language for hardware related routines and low level system interfaces. Assembler language is not accepted for applications of any kind since the cost of maintenance is too high;

(2) PASCAL for all nonmathematical applications, including the LSI-11 microprocessor;

(3) FORTRAN for mathematics programs. The interactive interface may be written in PASCAL while all mathematics oriented subroutines may be written in FORTRAN and then the two linked together.

The importance of having a well-known programming language cannot be stressed enough, since a number of applications at LEAR are written by visitors from all over Europe, whose education ranges from last-year undergraduate students to university professors. A student who comes for six months cannot be expected to produce a serious application program in such a short time if, in addition to solving his problem, he has to learn how to use the available tools.

\section{Conclusions}

The successful operation of a machine with working modes as complex as LEAR, with its associated experimental area, requires a powerful but still easy-to-use control system that has the facilities to enable machine physicists to adapt the machine to new conditions.

The existing control system provides the base for the developments, described above, planned to ensure that the accelerator will provide the beams required by the physics community in the future as successfully as now.

\section{References}

[1] U. Tallgren et al., The distributed computer control for the CERN Low Energy Ring (LEAR), presented at 6th IFAC Workshop on Distributed Computer Control Systems, Monterey, CA, USA (May 1985).

[2] Physics at LEAR with low energy cooled antiprotons, Proc. Workshop on Physics at LEAR with Low-Energy Cooled Antiprotons, May 9-16, 1982, Erice, S1cily, eds., U. Gastaldi and R. Klapisch. (Plenum, New York).

[3] D.J. Simon, D. Dumollard and K.G. Rensfelt, CERN/PS/85-13 (EA) (1985).

[4] J. Knott and R. Nettleton, these Proceedings (1985 Accelerator Controls Workshop) Nucl. Instr. and Meth. A247 (1986) 223.

[5] S. Hult, The LEAR GFA editor, PS/LEA/Note 84-6.

[6] E. Martensson, The LEAR Energy Scanning Editor, PS/LEA/Note 85-8 (1985).

[7] W. Hardt, Ultraslow ejection at LEAR, PS/DK/LEA Note 81-6 (1981).

[8] Design study of a facility for experiments with low energy antiprotons (LEAR), CERN/PS/DL 80-7 (May 1980).

[9] J. Jäger, Computer programs for the control of LEAR, PS/LEA/Note 83-1 (1983). 